\title{
On the $\Delta \Delta$ component of the deuteron in the Nambu-Jona-Lasinio model of light nuclei
}

\author{
A. N. Ivanov ${ }^{*}$, H. Oberhummer ${ }^{\dagger}$, N. I. Troitskaya ${ }^{\ddagger}$, M. Faber ${ }^{\S}$
}

November 11, 2018

Institut für Kernphysik, Technische Universität Wien,
Wiedner Hauptstr. 8-10, A-1040 Vienna, Austria

\begin{abstract}
The probability $P(\Delta \Delta)$ to find the $\Delta \Delta$ component inside the deuteron, where $\Delta$ stands for the $\Delta(1232)$ resonance, is calculated in the Nambu-Jona-Lasinio model of light nuclei. We obtain $P(\Delta \Delta)=0.3 \%$. This prediction agrees good with the experimental estimate $P(\Delta \Delta)<0.4 \%$ at $90 \%$ of CL (D. Allasia et al., Phys. Lett. B174 (1986) 450).
\end{abstract}

PACS: 11.10.Ef, 13.75.Cs, 14.20.Dh, 21.30.Fe

Keywords: field theory, QCD, deuteron, $\Delta$ isobar

*E-mail: ivanov@kph.tuwien.ac.at, Tel.: +43-1-58801-14261, Fax: +43-1-58801-14299

$\dagger$ E-mail: ohu@kph.tuwien.ac.at, Tel.: +43-1-58801-14251, Fax: +43-1-58801-14299

${ }_{\ddagger}^{\ddagger}$ Permanent Address: State Technical University, Department of Nuclear Physics, 195251 St. Petersburg, Russian Federation

${ }^{\S}$ E-mail: faber@kph.tuwien.ac.at, Tel.: +43-1-58801-14261, Fax: +43-1-58801-14299 


\section{Introduction}

As has been stated in Ref.[1] that nowadays there is a consensus concerning the existence of non-nucleonic degrees of freedom in nuclei. The non-nucleonic degrees of freedom can be described either within QCD in terms of quarks and gluons [2] or in terms of mesons and nucleon resonances [3].

In this letter we investigate the non-nucleonic degrees of freedom in terms of the $\Delta(1232)$ resonance and calculate the contribution of the $\Delta \Delta$ component to the deuteron in the Nambu-Jona-Lasinio model of light nuclei or differently the nuclear Nambu-JonaLasinio (NNJL) model [4,5]. As has been shown in Ref. [4] the NNJL model is motivated by QCD. The deuteron appears in the nuclear phase of $Q C D$ as a neutron-proton collective excitation, the Cooper np-pair, induced by a phenomenological local four-nucleon interaction. The NNJL model describes low-energy nuclear forces in terms of one-nucleon loop exchanges providing a minimal transfer of nucleon flavours from initial to final nuclear states and accounting for contributions of nucleon-loop anomalies which are completely determined by one-nucleon loop diagrams. The dominance of contributions of nucleonloop anomalies to effective Lagrangians of low-energy nuclear interactions is justified in the large $N_{C}$ expansion, where $N_{C}$ is the number of quark colours [4]. As has been shown in Refs. [5] the NNJL model describes good low-energy nuclear forces for electromagnetic and weak nuclear reactions with the deuteron of astrophysical interest such as the neutron-proton radiative capture $\mathrm{n}+\mathrm{p} \rightarrow \mathrm{D}+\gamma$, the solar proton burning $\mathrm{p}+\mathrm{p} \rightarrow \mathrm{D}$ $+\mathrm{e}^{+}+\nu_{\mathrm{e}}$, the pep-process $\mathrm{p}+\mathrm{e}^{-}+\mathrm{p} \rightarrow \mathrm{D}+\nu_{\mathrm{e}}$ and reactions of the disintegration of the deuteron by neutrinos and anti-neutrinos caused by charged $\nu_{\mathrm{e}}+\mathrm{D} \rightarrow \mathrm{e}^{-}+\mathrm{p}+\mathrm{p}$, $\bar{\nu}_{\mathrm{e}}+\mathrm{D} \rightarrow \mathrm{e}^{+}+\mathrm{n}+\mathrm{n}$ and neutral $\nu_{\mathrm{e}}\left(\bar{\nu}_{\mathrm{e}}\right)+\mathrm{D} \rightarrow \nu_{\mathrm{e}}\left(\bar{\nu}_{\mathrm{e}}\right)+\mathrm{n}+\mathrm{p}$ weak currents.

A phenomenological Lagrangian of the npD interaction is defined by [4]

$$
\mathcal{L}_{\mathrm{npD}}(x)=-i g_{\mathrm{V}}\left[\bar{p}(x) \gamma^{\mu} n^{c}(x)-\bar{n}(x) \gamma^{\mu} p^{c}(x)\right] D_{\mu}(x),
$$

where $D_{\mu}(x), n(x)$ and $p(x)$ are the interpolating fields of the deuteron, the neutron and the proton. The phenomenological coupling constant $g_{\mathrm{V}}$ is related to the electric quadrupole moment of the deuteron $Q_{\mathrm{D}}=0.286 \mathrm{fm}: g_{\mathrm{V}}^{2}=2 \pi^{2} Q_{\mathrm{D}} M_{\mathrm{N}}^{2}[4]$, where $M_{\mathrm{N}}=$ $940 \mathrm{MeV}$ is the nucleon mass. In the isotopically invariant form the phenomenological interaction Eq.(1.1) can be written as

$$
\mathcal{L}_{\mathrm{npD}}(x)=g_{\mathrm{V}} \bar{N}(x) \gamma^{\mu} \tau_{2} N^{c}(x) D_{\mu}(x),
$$

where $\tau_{2}$ is the Pauli isotopical matrix and $N(x)$ is a doublet of a nucleon field with components $N(x)=(p(x), n(x)), N^{c}(x)=C \bar{N}^{T}(x)$ and $\bar{N}^{c}(x)=N^{T}(x) C$, where $C$ is a charge conjugation matrix and $T$ is a transposition.

In the NNJL model [5] the $\Delta(1232)$ resonance is the Rarita-Schwinger field [6] $\Delta_{\mu}^{a}(x)$, the isotopical index $a$ runs over $a=1,2,3$, having the following free Lagrangian $[7,8]$ :

$$
\mathcal{L}_{\text {kin }}^{\Delta}(x)=\bar{\Delta}_{\mu}^{a}(x)\left[-\left(i \gamma^{\alpha} \partial_{\alpha}-M_{\Delta}\right) g^{\mu \nu}+\frac{1}{4} \gamma^{\mu} \gamma^{\beta}\left(i \gamma^{\alpha} \partial_{\alpha}-M_{\Delta}\right) \gamma_{\beta} \gamma^{\nu}\right] \Delta_{\nu}^{a}(x),
$$

where $M_{\Delta}=1232 \mathrm{MeV}$ is the mass of the $\Delta(1232)$ resonance field $\Delta_{\mu}^{a}(x)$. In terms of the 
eigenstates of the electric charge operator the fields $\Delta_{\mu}^{a}(x)$ are given by $[7,8]$

$$
\begin{gathered}
\Delta_{\mu}^{1}(x)=\frac{1}{\sqrt{2}}\left(\begin{array}{c}
\Delta_{\mu}^{++}(x)-\Delta_{\mu}^{0}(x) / \sqrt{3} \\
\Delta_{\mu}^{+}(x) / \sqrt{3}-\Delta_{\mu}^{-}(x)
\end{array}\right), \Delta_{\mu}^{2}(x)=\frac{i}{\sqrt{2}}\left(\begin{array}{c}
\Delta_{\mu}^{++}(x)+\Delta_{\mu}^{0}(x) / \sqrt{3} \\
\Delta_{\mu}^{+}(x) / \sqrt{3}+\Delta_{\mu}^{-}(x)
\end{array}\right), \\
\Delta_{\mu}^{3}(x)=-\sqrt{\frac{2}{3}}\left(\begin{array}{c}
\Delta_{\mu}^{+}(x) \\
\Delta_{\mu}^{0}(x)
\end{array}\right) .
\end{gathered}
$$

The fields $\Delta_{\mu}^{a}(x)$ obey the subsidiary constraints: $\partial^{\mu} \Delta_{\mu}^{a}(x)=\gamma^{\mu} \Delta_{\mu}^{a}(x)=0$ [7-9]. The Green function of the free $\Delta$-field is determined by

$$
<0\left|\mathrm{~T}\left(\Delta_{\mu}\left(x_{1}\right) \bar{\Delta}_{\nu}\left(x_{2}\right)\right)\right| 0>=-i S_{\mu \nu}\left(x_{1}-x_{2}\right)
$$

In the momentum representation $S_{\mu \nu}(x)$ reads [5-8]:

$$
S_{\mu \nu}(p)=\frac{1}{M_{\Delta}-\hat{p}}\left(-g_{\mu \nu}+\frac{1}{3} \gamma_{\mu} \gamma_{\nu}+\frac{1}{3} \frac{\gamma_{\mu} p_{\nu}-\gamma_{\nu} p_{\mu}}{M_{\Delta}}+\frac{2}{3} \frac{p_{\mu} p_{\nu}}{M_{\Delta}^{2}}\right)
$$

The most general form of the $\pi \mathrm{N} \Delta$ interaction compatible with the requirements of chiral symmetry reads [7]:

$$
\begin{aligned}
& \mathcal{L}_{\pi \mathrm{N} \Delta}(x)=\frac{g_{\pi \mathrm{N} \Delta}}{2 M_{\mathrm{N}}} \bar{\Delta}_{\omega}^{a}(x) \Theta^{\omega \varphi} N(x) \partial_{\varphi} \pi^{a}(x)+\text { h.c. }= \\
& =\frac{g_{\pi \mathrm{N} \Delta}}{\sqrt{6} M_{\mathrm{N}}}\left[\frac{1}{\sqrt{2}} \bar{\Delta}_{\omega}^{+}(x) \Theta^{\omega \varphi} n(x) \partial_{\varphi} \pi^{+}(x)-\frac{1}{\sqrt{2}} \bar{\Delta}_{\omega}^{0}(x) \Theta^{\omega \varphi} p(x) \partial_{\varphi} \pi^{-}(x)\right. \\
& \left.-\bar{\Delta}_{\omega}^{+}(x) \Theta^{\omega \varphi} p(x) \partial_{\varphi} \pi^{0}(x)-\bar{\Delta}_{\omega}^{0}(x) \Theta^{\omega \varphi} p(x) \partial_{\varphi} \pi^{0}(x)+\ldots\right]
\end{aligned}
$$

where $\pi^{a}(x)$ is the pion field with the components $\pi^{1}(x)=\left(\pi^{-}(x)+\pi^{+}(x)\right) / \sqrt{2}, \pi^{2}(x)=$ $\left(\pi^{-}(x)-\pi^{+}(x)\right) / i \sqrt{2}$ and $\pi^{3}(x)=\pi^{0}(x)$. The tensor $\Theta^{\omega \varphi}$ is given in Ref. [7]: $\Theta^{\omega \varphi}=$ $g^{\omega \varphi}-(Z+1 / 2) \gamma^{\omega} \gamma^{\varphi}$, where the parameter $Z$ is arbitrary. The parameter $Z$ defines the $\pi \mathrm{N} \Delta$ coupling off-mass shell of the $\Delta(1232)$ resonance. There is no consensus on the exact value of $Z$. From theoretical point of view $Z=1 / 2$ is preferred [7]. Phenomenological studies give only the bound $|Z| \leq 1 / 2[9]$. The value of the coupling constant $g_{\pi \mathrm{N} \Delta}$ relative to the coupling constant $g_{\pi \mathrm{NN}}$ is $g_{\pi \mathrm{N} \Delta}=2 g_{\pi \mathrm{NN}}$ [10]. As has been shown in Ref. [5] for the description of the experimental value of the cross section for the neutron-proton radiative capture for thermal neutrons the parameter $Z$ should be equal to $Z=0.473$. This agrees with the experimental bound [9]. At $Z=1 / 2$ we get the result agreeing with the experimental value of the cross section for the neutron-proton radiative capture with accuracy about $3 \%[5]$.

For the subsequent calculations of the $\Delta \Delta$ component of the deuteron it is useful to have the Lagrangian of the $\pi \mathrm{N} \Delta$ interaction taken in the equivalent form

$$
\mathcal{L}_{\pi \mathrm{N} \Delta}(x)=\frac{g_{\pi \mathrm{N} \Delta}}{2 M_{\mathrm{N}}} \partial_{\varphi} \pi^{a}(x) \bar{N}^{c}(x) \Theta^{\varphi \omega} \Delta_{\omega}^{a}(x)^{c}+\text { h.c. },
$$

where $\Delta_{\omega}^{a}(x)^{c}=C \bar{\Delta}_{\omega}^{a}(x)^{T}$. Now we can proceed to the evaluation of the $\Delta \Delta$ component of the deuteron. 


\section{Effective $\Delta \Delta \mathrm{D}$ interaction}

In the NNJL model the existence of the $\Delta \Delta$ component of the deuteron we can understand in terms of the coupling constants of the effective $\Delta \Delta \mathrm{D}$ interaction.

In order to evaluate the Lagrangian of the effective $\Delta \Delta \mathrm{D}$ interaction $\mathcal{L}_{\text {eff }}^{\Delta \Delta \mathrm{D}}(x)$ we have to obtain, first, the effective Lagrangian of the transition $\mathrm{N}+\mathrm{N} \rightarrow \Delta+\Delta$. This effective Lagrangian we define in the one-pion exchange approximation $[5,11]$

$$
\begin{aligned}
\int d^{4} x \mathcal{L}_{\mathrm{eff}}^{\mathrm{NN} \rightarrow \Delta \Delta}(x)= & -\frac{g_{\pi \mathrm{N} \Delta}^{2}}{8 M_{\mathrm{N}}^{2}} \iint d^{4} x_{1} d^{4} x_{2}\left[\bar{\Delta}_{\alpha}^{a}\left(x_{1}\right) \Theta^{\alpha \beta} N\left(x_{1}\right)\right] \\
& \times \frac{\partial}{\partial x_{1}^{\beta}} \frac{\partial}{\partial x_{1}^{\varphi}}\left[\delta^{a b} \Delta\left(x_{1}-x_{2}\right)\right]\left[\bar{N}^{c}\left(x_{2}\right) \Theta^{\varphi \omega} \Delta_{\omega}^{b}\left(x_{2}\right)^{c}\right],
\end{aligned}
$$

where $\Delta\left(x_{1}-x_{2}\right)$ is the Green function of $\pi$-mesons. In terms of the Lagrangians of the npD interaction and the $\mathrm{N}+\mathrm{N} \rightarrow \Delta+\Delta$ transition the Lagrangian of the effective $\Delta \Delta \mathrm{D}$ interaction can be defined by

$$
\begin{aligned}
& \int d^{4} x \mathcal{L}_{\mathrm{eff}}^{\Delta \Delta \mathrm{D}}(x)=-i g_{\mathrm{V}} \frac{g_{\pi \mathrm{N} \Delta}^{2}}{4 M_{\mathrm{N}}^{2}} \int d^{4} x d^{4} x_{1} d^{4} x_{2} D_{\mu}(x) \\
& {\left[\bar{\Delta}_{\alpha}^{a}\left(x_{1}\right) \Theta^{\alpha \beta} S_{F}\left(x-x_{1}\right) \gamma^{\mu} \tau_{2} S_{F}^{c}\left(x-x_{2}\right) \Theta^{\varphi \omega} \Delta_{\omega}^{a}\left(x_{2}\right)^{c}\right] \frac{\partial}{\partial x_{1}^{\beta}} \frac{\partial}{\partial x_{1}^{\varphi}} \Delta\left(x_{1}-x_{2}\right),}
\end{aligned}
$$

where $S_{F}\left(x-x_{1}\right)$ and $S_{F}^{c}\left(x-x_{2}\right)$ are the Green functions of the free nucleon and antinucleon fields, respectively.

Such a definition of the contribution of the $\Delta \Delta$ component to the deuteron is in agreement with that given by Niephaus et al. [12] in the potential model approach (PMA).

For the evaluation of the effective Lagrangian $\mathcal{L}_{\text {eff }}^{\Delta \Delta \mathrm{D}}(x)$ we would follow the large $N_{C}$ expansion approach to non-perturbative QCD [4]. In the large $N_{C}$ approach to non-perturbative QCD with $S U\left(N_{C}\right)$ gauge group at $N_{C} \rightarrow \infty$ the nucleon mass is proportional to the number of quark colour degrees of freedom, $M_{\mathrm{N}} \sim N_{C}$ [13]. It is well-known that for the evaluation of effective Lagrangians all momenta of interacting particles should be kept off-mass shell. This implies that at leading order in the large $N_{C}$ expansion corresponding the $1 / M_{\mathrm{N}}$ expansion of the momentum integral defining the effective Lagrangian $\mathcal{L}_{\text {eff }}^{\Delta \Delta \mathrm{D}}(x)$ one can neglect the momenta of interacting particles with respect to the mass of virtual nucleons. As a result the effective Lagrangian $\mathcal{L}_{\text {eff }}^{\Delta \Delta \mathrm{D}}(x)$ reduces itself to the local form and reads

$$
\mathcal{L}_{\text {eff }}^{\Delta \Delta \mathrm{D}}(x)=\frac{g_{\mathrm{V}}}{16 \pi^{2}} \frac{g_{\pi \mathrm{N} \Delta}^{2}}{4 M_{\mathrm{N}}^{2}}\left[\bar{\Delta}_{\alpha}^{a}(x) \Theta^{\alpha \mu \omega} \tau_{2} \Delta_{\omega}^{a}(x)^{c}\right] D_{\mu}(x),
$$

where the structure function $\Theta^{\alpha \mu \omega}$ is given by the momentum integral

$$
\Theta^{\alpha \mu \omega}=\int \frac{d^{4} k}{\pi^{2} i} \frac{1}{M_{\pi}^{2}-k^{2}} \Theta^{\alpha \beta} k_{\beta} \frac{1}{M_{\mathrm{N}}-\hat{k}} \gamma^{\mu} \frac{1}{M_{\mathrm{N}}+\hat{k}} k_{\varphi} \Theta^{\varphi \omega} .
$$

Integrating over $k$ we obtain

$$
\begin{aligned}
\Theta^{\alpha \mu \omega} & =\frac{1}{3}\left[I_{1}\left(M_{\mathrm{N}}\right)-\frac{5}{2} M_{\mathrm{N}}^{2} I_{2}\left(M_{\mathrm{N}}\right)\right] \Theta^{\alpha \beta} \gamma^{\mu} \Theta_{\beta}{ }^{\omega} \\
& -\frac{1}{12}\left[I_{1}\left(M_{\mathrm{N}}\right)-M_{\mathrm{N}}^{2} I_{2}\left(M_{\mathrm{N}}\right)\right]\left(\Theta^{\alpha \beta} \gamma_{\beta} \Theta^{\mu \omega}+\Theta^{\alpha \mu} \gamma_{\varphi} \Theta^{\varphi \omega}\right),
\end{aligned}
$$


where the quadratically, $I_{1}\left(M_{\mathrm{N}}\right)$, and logarithmically, $I_{2}\left(M_{\mathrm{N}}\right)$, divergent integrals are determined by [4]

$$
\begin{aligned}
& I_{1}\left(M_{\mathrm{N}}\right)=\int \frac{d^{4} k}{\pi^{2} i} \frac{1}{M_{\mathrm{N}}^{2}-k^{2}}=2\left[\Lambda \sqrt{M_{\mathrm{N}}^{2}+\Lambda^{2}}-M_{\mathrm{N}}^{2} \ln \left(\frac{\Lambda}{M_{\mathrm{N}}}+\sqrt{1+\frac{\Lambda^{2}}{M_{\mathrm{N}}^{2}}}\right)\right], \\
& I_{2}\left(M_{\mathrm{N}}\right)=\int \frac{d^{4} k}{\pi^{2} i} \frac{1}{\left(M_{\mathrm{N}}^{2}-k^{2}\right)^{2}}=2\left[\ln \left(\frac{\Lambda}{M_{\mathrm{N}}}+\sqrt{1+\frac{\Lambda^{2}}{M_{\mathrm{N}}^{2}}}\right)-\frac{\Lambda}{\sqrt{M_{\mathrm{N}}^{2}+\Lambda^{2}}}\right] .
\end{aligned}
$$

The cut-off $\Lambda$ restricts from above $3-$ momenta of fluctuating nucleon fields. Since we have no closed nucleon loops, the cut-off $\Lambda$ cannot be determined by the scale of the deuteron size $r_{\mathrm{D}} \sim 1 / \Lambda_{\mathrm{D}}$ [4]. The natural value of $\Lambda$ is the scale of the Compton wavelength of the nucleon $\lambda_{\mathrm{N}}=1 / M_{\mathrm{N}}=0.21 \mathrm{fm}$, i.e. $\Lambda=M_{\mathrm{N}}$.

The Lagrangian $\mathcal{L}_{\text {eff }}^{\Delta \Delta \mathrm{D}}(x)$ of the effective $\Delta \Delta \mathrm{D}$ interaction we obtain in the form

$$
\begin{aligned}
& \mathcal{L}_{\text {eff }}^{\Delta \Delta}(x)=g_{\Delta \Delta \mathrm{D}}\left[\bar{\Delta}_{\alpha}^{a}(x) \Theta^{\alpha \beta} \gamma^{\mu} \Theta_{\beta}{ }^{\omega} \tau_{2} \Delta_{\omega}^{a}(x)^{c}\right] D_{\mu}(x) \\
& +\bar{g}_{\Delta \Delta \mathrm{D}}\left[\bar{\Delta}_{\alpha}^{a}(x)\left(\Theta^{\alpha \beta} \gamma_{\beta} \Theta^{\mu \omega}+\Theta^{\alpha \mu} \gamma_{\varphi} \Theta^{\varphi \omega}\right) \tau_{2} \Delta_{\omega}^{a}(x)^{c}\right] D_{\mu}(x)= \\
& =-i g_{\Delta \Delta \mathrm{D}}\left[\bar{\Delta}_{\alpha}^{-}(x) \Theta^{\alpha \beta} \gamma^{\mu} \Theta_{\beta}{ }^{\omega} \Delta_{\omega}^{++}(x)^{c}-\bar{\Delta}_{\alpha}^{++}(x) \Theta^{\alpha \beta} \gamma^{\mu} \Theta_{\beta}{ }^{\omega} \Delta_{\omega}^{-}(x)^{c}\right. \\
& \left.+\bar{\Delta}_{\alpha}^{+}(x) \Theta^{\alpha \beta} \gamma^{\mu} \Theta_{\beta}{ }^{\omega} \Delta_{\omega}^{0}(x)^{c}-\bar{\Delta}_{\alpha}^{0}(x) \Theta^{\alpha \beta} \gamma^{\mu} \Theta_{\beta}{ }^{\omega} \Delta_{\omega}^{+}(x)^{c}\right] D_{\mu}(x) \\
& -i \bar{g}_{\Delta \Delta \mathrm{D}}\left[\bar{\Delta}_{\alpha}^{-}(x)\left(\Theta^{\alpha \beta} \gamma_{\beta} \Theta^{\mu \omega}+\Theta^{\alpha \mu} \gamma_{\varphi} \Theta^{\varphi \omega}\right) \Delta_{\omega}^{++}(x)^{c}\right. \\
& -\bar{\Delta}_{\alpha}^{++}(x)\left(\Theta^{\alpha \beta} \gamma_{\beta} \Theta^{\mu \omega}+\Theta^{\alpha \mu} \gamma_{\varphi} \Theta^{\varphi \omega}\right) \Delta_{\omega}^{-}(x)^{c} \\
& +\bar{\Delta}_{\alpha}^{+}(x)\left(\Theta^{\alpha \beta} \gamma_{\beta} \Theta^{\mu \omega}+\Theta^{\alpha \mu} \gamma_{\varphi} \Theta^{\varphi \omega}\right) \Delta_{\omega}^{0}(x)^{c} \\
& \left.-\bar{\Delta}_{\alpha}^{0}(x)\left(\Theta^{\alpha \beta} \gamma_{\beta} \Theta^{\mu \omega}+\Theta^{\alpha \mu} \gamma_{\varphi} \Theta^{\varphi \omega}\right) \Delta_{\omega}^{+}(x)^{c}\right],
\end{aligned}
$$

where the effective coupling constants $g_{\Delta \Delta \mathrm{D}}$ and $\bar{g}_{\Delta \Delta \mathrm{D}}$ read

$$
\begin{aligned}
& g_{\Delta \Delta \mathrm{D}}=g_{\mathrm{V}} \frac{7 g_{\pi \mathrm{N} \Delta}^{2}}{384 \pi^{2}}\left[\frac{\Lambda}{\sqrt{M_{\mathrm{N}}^{2}+\Lambda^{2}}}\left(1+\frac{2}{7} \frac{\Lambda^{2}}{M_{\mathrm{N}}^{2}}\right)-\ln \left(\frac{\Lambda}{M_{\mathrm{N}}}+\sqrt{1+\frac{\Lambda^{2}}{M_{\mathrm{N}}^{2}}}\right)\right] \\
& \bar{g}_{\Delta \Delta \mathrm{D}}=-g_{\mathrm{V}} \frac{g_{\pi \mathrm{N} \Delta}^{2}}{192 \pi^{2}}\left[\frac{\Lambda}{\sqrt{M_{\mathrm{N}}^{2}+\Lambda^{2}}}\left(1+\frac{1}{2} \frac{\Lambda^{2}}{M_{\mathrm{N}}^{2}}\right)-\ln \left(\frac{\Lambda}{M_{\mathrm{N}}}+\sqrt{1+\frac{\Lambda^{2}}{M_{\mathrm{N}}^{2}}}\right)\right] .
\end{aligned}
$$

On-mass shell of the $\Delta(1232)$ resonance, i.e. in the case of the PMA [1,12], the contribution of the parameter $Z$ vanishes and the effective $\Delta \Delta \mathrm{D}$ interaction acquires the form

$$
\begin{aligned}
& \mathcal{L}_{\text {eff }}^{\Delta \Delta \mathrm{D}}(x)=g_{\Delta \Delta \mathrm{D}} g^{\alpha \beta}\left[\bar{\Delta}_{\alpha}^{a}(x) \gamma^{\mu} \tau_{2} \Delta_{\beta}^{a}(x)^{c}\right] D_{\mu}(x)= \\
& =-i g_{\Delta \Delta \mathrm{D}} g^{\alpha \beta}\left[\bar{\Delta}_{\alpha}^{-}(x) \gamma^{\mu} \Delta_{\beta}^{++}(x)^{c}-\bar{\Delta}_{\alpha}^{++}(x) \gamma^{\mu} \Delta_{\beta}^{-}(x)^{c}\right. \\
& \left.+\bar{\Delta}_{\alpha}^{+}(x) \gamma^{\mu} \Delta_{\beta}^{0}(x)^{c}-\bar{\Delta}_{\alpha}^{0}(x) \gamma^{\mu} \Delta_{\beta}^{+}(x)^{c}\right] D_{\mu}(x) .
\end{aligned}
$$

The total probability $P(\Delta \Delta)$ to find the $\Delta \Delta$ component inside the deuteron we determine as follows

$$
P(\Delta \Delta)=\frac{d \Gamma(\mathrm{D} \rightarrow \Delta \Delta)}{d \Gamma(\mathrm{D} \rightarrow \mathrm{np})}
$$


where $d \Gamma(\mathrm{D} \rightarrow \Delta \Delta)$ and $d \Gamma(\mathrm{D} \rightarrow \mathrm{np})$ are the differential rates of the transitions $\mathrm{D} \rightarrow \Delta$ $+\Delta$ and $\mathrm{D} \rightarrow \mathrm{n}+\mathrm{p}$, respectively, defined by

$$
\begin{aligned}
& d \Gamma\left(\mathrm{D}(P) \rightarrow \Delta\left(p_{1}\right) \Delta\left(p_{2}\right)\right)=8 g_{\Delta \Delta \mathrm{D}}^{2} \frac{d \Phi_{\Delta \Delta}\left(p_{1}, p_{2}\right)}{6 \sqrt{s}}\left(-g_{\mu \nu}+\frac{P_{\mu} P_{\nu}}{s}\right) \\
& \times \operatorname{tr}\left\{\left(M_{\Delta}+\hat{p}_{1}\right)\left(-g_{\alpha \beta}+\frac{1}{3} \gamma_{\alpha} \gamma_{\beta}+\frac{1}{3} \frac{\gamma_{\alpha} p_{1 \beta}-\gamma_{\beta} p_{1 \alpha}}{M_{\Delta}}+\frac{2}{3} \frac{p_{1 \alpha} p_{1 \beta}}{M_{\Delta}^{2}}\right) \gamma^{\mu}\right. \\
& \left.\times\left(-g^{\alpha \beta}+\frac{1}{3} \gamma^{\beta} \gamma^{\alpha}+\frac{1}{3} \frac{\gamma^{\beta} p_{2}^{\alpha}-\gamma^{\alpha} p_{2}^{\beta}}{M_{\Delta}}+\frac{2}{3} \frac{p_{2}^{\beta} p_{2}^{\alpha}}{M_{\Delta}^{2}}\right)\left(-M_{\Delta}+\hat{p}_{2}\right) \gamma^{\nu}\right\}, \\
& d \Gamma\left(\mathrm{D}(P) \rightarrow \mathrm{n}\left(p_{1}\right) \mathrm{p}\left(p_{2}\right)\right)=4 g_{\mathrm{V}}^{2} \frac{d \Phi_{\mathrm{np}}\left(p_{1}, p_{2}\right)}{6 \sqrt{s}}\left(-g_{\mu \nu}+\frac{P_{\mu} P_{\nu}}{s}\right) \\
& \times \operatorname{tr}\left\{\left(M_{\mathrm{N}}+\hat{p}_{1}\right) \gamma^{\mu}\left(-M_{\mathrm{N}}+\hat{p}_{2}\right) \gamma^{\nu}\right\} .
\end{aligned}
$$

We have denoted as $P=p_{1}+p_{2}$ and $P^{2}=s$ the 4 -momentum and the invariant squared mass of the deuteron, respectively. Then, $d \Phi_{\Delta \Delta}\left(p_{1}, p_{2}\right)$ and $d \Phi_{\mathrm{np}}\left(p_{1}, p_{2}\right)$ are the phase volumes of the $\Delta \Delta$ and np states. The two-particle phase volume is equal to

$$
d \Phi\left(p_{1}, p_{2}\right)=(2 \pi)^{4}\left(P-p_{1}-p_{2}\right) \frac{d^{3} p_{1}}{(2 \pi)^{3} 2 E_{1}} \frac{d^{3} p_{2}}{(2 \pi)^{3} 2 E_{2}}
$$

At leading order in the large $N_{C}$ expansion, when we can neglect the mass difference between the $\Delta(1232)$ resonance and the nucleon, the phase volumes $d \Phi_{\Delta \Delta}\left(p_{1}, p_{2}\right)$ and $d \Phi_{\mathrm{np}}\left(p_{1}, p_{2}\right)$ are equal

$$
d \Phi_{\Delta \Delta}\left(p_{1}, p_{2}\right)=d \Phi_{\mathrm{np}}\left(p_{1}, p_{2}\right)=d \Phi\left(p_{1}, p_{2}\right) .
$$

The differential rates $d \Gamma\left(\mathrm{D}(P) \rightarrow \Delta\left(p_{1}\right) \Delta\left(p_{2}\right)\right)$ and $d \Gamma\left(\mathrm{D}(P) \rightarrow \mathrm{n}\left(p_{1}\right) \mathrm{p}\left(p_{2}\right)\right)$ calculated at leading order in the large $N_{C}$ expansion are given by

$$
\begin{aligned}
d \Gamma\left(\mathrm{D}(P) \rightarrow \Delta\left(p_{1}\right) \Delta\left(p_{2}\right)\right) & =\frac{10}{9} \times 8 \times g_{\Delta \Delta \mathrm{D}}^{2} \times \sqrt{s} d \Phi\left(p_{1}, p_{2}\right), \\
d \Gamma\left(\mathrm{D}(P) \rightarrow \mathrm{n}\left(p_{1}\right) \mathrm{p}\left(p_{2}\right)\right) & =4 \times g_{\mathrm{V}}^{2} \times \sqrt{s} d \Phi\left(p_{1}, p_{2}\right) .
\end{aligned}
$$

Hence, the probability $P(\Delta \Delta)$ to find the $\Delta \Delta$ component inside the deuteron amounts to

$$
P(\Delta \Delta)=\frac{10}{9} \times \frac{2 g_{\Delta \Delta \mathrm{D}}^{2}}{g_{\mathrm{V}}^{2}}=0.3 \%,
$$

where the numerical value is obtained at $\Lambda=M_{\mathrm{N}}$.

Our theoretical prediction agrees good with recent experimental estimate of the upper limit $P(\Delta \Delta)<0.4 \%$ at $90 \%$ of CL [14] quoted by Dymarz and Khanna [1].

\section{Conclusion}

The theoretical estimate of the contribution of the $\Delta \Delta$ component to the deuteron obtained in the NNJL model agrees good with the experimental upper limit. Indeed, for 
the $\Delta(1232)$ resonance on-mass shell $[1,12]$ we predict $P(\Delta \Delta)=0.3 \%$ whereas experimentally $P(\Delta \Delta)$ is restricted by $P(\Delta \Delta)<0.4 \%$ at $90 \%$ of CL [14].

Off-mass shell of the $\Delta(1232)$ resonance, where the parameter $Z$ should contribute, our prediction for $P(\Delta \Delta)$ can be changed, of course. Moreover, due to $Z$ dependence the contributions of the $\Delta \Delta$ component to amplitudes of different low-energy nuclear reactions and physical quantities can differ each other. However, we would like to emphasize that in the NNJL model by using the effective $\Delta \Delta \mathrm{D}$ interaction determined by Eq.(2.7) one can calculate the contribution of the $\Delta \Delta$ component of the deuteron to the amplitude of any low-energy nuclear reaction with the deuteron in the initial or final state.

In our approach we do not distinguish contributions of the $\Delta \Delta$-pair with a definite orbital momentum ${ }^{3} \mathrm{~S}_{1}^{\Delta \Delta},{ }^{3} \mathrm{D}_{1}^{\Delta \Delta}$ and so on to the effective $\Delta \Delta \mathrm{D}$ interaction Eq.(2.7). The obtained value of the probability $P(\Delta \Delta)$ should be considered as a sum of all possible states with a certain orbital momentum.

Our prediction $P(\Delta \Delta)=0.3 \%$ agrees reasonably well with the result obtained by Dymarz and Khanna in the PMA [1]: $P(\Delta \Delta) \simeq 0.4 \div 0.5 \%$. Unlike our approach Dymarz and Khanna have given a percentage of the probabilities of different states ${ }^{3} \mathrm{~S}_{1}^{\Delta \Delta},{ }^{3} \mathrm{D}_{1}^{\Delta \Delta}$ and so to the wave function of the deuteron. In our approach the deuteron couples to itself and other particles through the one-baryon loop exchanges. The effective Lagrangian $\mathcal{L}_{\text {eff }}^{\Delta \Delta \mathrm{D}}(x)$ of the $\Delta \Delta \mathrm{D}$ interaction given by Eq.(2.7) defines completely the contribution of the $\Delta \Delta$ intermediate states to baryon-loop exchanges. The decomposition of the effective $\Delta \Delta \mathrm{D}$ interaction in terms of the $\Delta \Delta$ states with a certain orbital momentum should violate Lorentz invariance for the evaluation of the contribution of every state to whether the amplitude of a low-energy nuclear reaction or a low-energy physical quantity. In the NNJL model this can lead to incorrect results. The relativistically covariant procedure of the decomposition of the interactions like the $\Delta \Delta \mathrm{D}$ one in terms of the states with a certain orbital momenta is now in progress in the NNJL model. However, a smallness of the contribution of the $\Delta \Delta$ component to the deuteron obtained in the NNJL model makes such a decomposition applied to the $\Delta \Delta \mathrm{D}$ interaction meaningless to some extent due to impossibility to measure the terms separately.

\section{Acknowledgement}

We are grateful to Prof. W. Plessas for discussions which stimulated this investigation. 


\section{References}

[1] R. Dymarz and F. C. Khanna, Nucl. Phys. A 516, 549 (1990).

[2] QUARKS AND NUCLEI, ed W. Weise, World Scientific, Singapore, 1989.

[3] MESONS IN NUCLEI, ed. M. Rho and D. H. Wilkinson, Noth-Holland, Amsterdam, 1979.

[4] A. N. Ivanov, H. Oberhummer, N. I. Troitskaya and M. Faber, Eur. Phys. J. 7, 519 (2000).

[5] A. N. Ivanov, H. Oberhummer, N. I. Troitskaya and M. Faber, Dynamics of lowenergy nuclear forces for electromagnetic and weak reactions with the deuteron in the Nambu-Jona-Lasinio model of light nuclei, Eur. Phys. J. A (in press).

[6] W. Rarita and J. Schwinger, Phys. Rev. 60, 61 (1941).

[7] L. M. Nath, B. Etemadi and J. D. Kimel, Phys. Rev. D 3, 2153 (1971).

[8] J. Kambor, The $\Delta(1232)$ as an Effective Degree of Freedom in Chiral Perturbation Theory, Talk given at the Workshop on Chiral Dynamics, 1997 Mainz, Germany, September 1-5, 1997; hep-ph/9711484 26 November 1997.

[9] K. Kabir, T. K. Dutta, Muslema Pervin and L. M. Nath, The Role of $\Delta(1232)$ in Two-pion Exchange Three-nucleon Potential, hep-th/9910043, October 1999.

[10] A. N. Ivanov, M. Nagy and N. I. Troitskaya, Phys. Rev. C 59, 451 (1999).

[11] D. O. Riska and G. E. Brown, Phys. Lett. B 38, 193 (1972); T.-S. Park, D.-P. Min and M. Rho, Phys. Rev. Lett. 74, 4153 (1995); Nucl. Phys. A 596, 515 (1996).

[12] G. H. Niephaus, M. Gari and B. Sommer, Phys. Rev. C 20, 1096 (1979).

[13] E. Witten, Nucl. Phys. 160, 59 (1979).

[14] D. Allasia et al., Phys. Lett. B 174, 450 (1986). 